\title{
Tip110/SART3 regulates IL-8 expression and 1 cosemate predicts the clinical outcomes in melanoma
}

\author{
Khalid Amine Timani ${ }^{* *}$, Balázs Győrffy²,3, Ying Liu' ${ }^{1}$, Khalid S. Mohammad ${ }^{4}$ and Johnny J. He ${ }^{1}$
}

\begin{abstract}
Tip110, an important regulator of several oncogenic proteins, was significantly downregulated in human metastatic melanoma cells exposed to a hypoxic condition. Therefore, in this study, we set to determine whether differential expression of Tip110 could be an important indicator for melanoma tumorigenesis and metastasis. We found that in melanoma, but not in other cancer types, Tip110 knockdown enhanced significant expression and secretion of IL-8 and melanoma cells invasions. This induction was further potentiated under hypoxia and by inflammatory cytokine and found independent of TNF-a autocrine signaling. We further showed that Tip110 knockdownmediated IL-8 induction involved IL-8 mRNA stability. Furthermore, the transcriptomic profiling data and survival from 455 melanoma patients demonstrated that the correlation between Tip110 expression and the clinical outcomes in melanoma was stage-dependent. These findings uncover important roles of Tip110 in melanoma tumorigenesis and metastasis through regulation of IL-8 and hope to provide new clues for future therapeutic strategies.
\end{abstract}

Keywords: Tip110/SART3, IL-8, Melanoma, Hypoxia, TP53, NF-kB

\section{Main text}

Malignant melanoma is an aggressive form of skin cancer and one of the leading causes of death in the United States. The demonstration of an increased incidence of metastasis at sites of inflammation supports the important role of inflammatory cytokines in the pathogenesis of tumor growth and metastasis. Among these cytokines is interleukin-8 (IL-8), which is well documented for its important roles in melanoma tumorigenesis and metastasis [1]. IL- 8 is normally induced and secreted by a diverse range of cells in response to pathologic stresses. Its serum levels in patients are significantly elevated and are correlated with advanced disease stage and poor prognosis. Thus, identification of the molecular players regulating IL-8, and in return promoting melanoma initiation and progression, will provide new clues for possible therapeutic strategies against melanoma.

HIV-1 Tat-interacting protein (Tip110), also known as squamous cell carcinoma antigen recognized by $\mathrm{T}$ cells 3 (SART3), is a multifaceted nuclear protein and has been shown to function in tumor antigenicity, regulation of gene transcription, mRNA synthesis, stem cell proliferation and differentiation, and embryogenesis [2]. We and others have reported that Tip110 interacts with and/or regulates several oncogenic proteins [2]. The high expression level of Tip110 has been found in a number of malignant tumor cell lines and cancerous tissues [2]. However, exposure of highly metastatic melanoma cells to hypoxia led to a significant downregulation of Tip110 together with TP53 in both in vitro and in a melanoma cancer bone metastasis mouse model [3]. Also, Tip110 expression was found to be dysregulated and correlated with a metastatic phenotype in melanoma and lung adenocarcinoma $[4,5]$. Therefore, it is plausible that differential expression of Tip110 could be an indicator for melanoma tumorigenesis.

Here, we demonstrated that Tip110 contributed to the regulation of the expression of IL-8 in melanoma cells and that the expression level of Tip110 had a prognostic value for melanoma patients. Furthermore, we identified the potential mechanism underlying the Tip110 knockdown-induced IL-8 expression.

\footnotetext{
* Correspondence: khalid.timani@unthsc.edu

${ }^{1}$ Department of Microbiology, Immunology, and Genetics, University of North Texas Health Science Center, 3500 Camp Bowie Blvd, Fort Worth, TX 76107, USA

Full list of author information is available at the end of the article
}

(c) The Author(s). 2018 Open Access This article is distributed under the terms of the Creative Commons Attribution 4.0 International License (http://creativecommons.org/licenses/by/4.0/), which permits unrestricted use, distribution, and reproduction in any medium, provided you give appropriate credit to the original author(s) and the source, provide a link to the Creative Commons license, and indicate if changes were made. The Creative Commons Public Domain Dedication waiver (http://creativecommons.org/publicdomain/zero/1.0/) applies to the data made available in this article, unless otherwise stated. 


\section{Results and discussion}

Tip110 knockdown induced IL-8 expression in melanoma with and without stimuli

Many alterations in tumor cells have been implicated as contributing factors to the tumor progression and metastasis. These changes include phenotypic characteristics such as autocrine expression of cytokines/growth factors and oncogenes or inactivation of tumor suppressor genes and loss of responsiveness to inhibitory cytokines/growth factors. Several reports had shown that human melanoma cells grown in cultures express and secrete numerous cytokines/growth factors either constitutively or upon induction [6]. In addition, our previous studies have shown a significant reduction of Tip110 together with TP53 expression in melanoma cells exposed to a hypoxic condition [3]. Therefore, we examined the expression of selected inflammatory cytokines and growth factors that are important during melanoma progression, metastasis, and survival, in the context of limited Tip110 expression [6]. Since TNF- $\alpha$ is involved in the biosynthesis of many other cytokines, we speculated that TNF- $\alpha$ treatment would potentiate any effect that could be exerted by Tip110 knockdown. The results showed that Tip110 knockdown led to significant up-regulation of both IL- 8 and TNF- $\alpha$ mRNA with and without TNF- $\alpha$ treatment (Fig. 1a). A moderate increase was found with IL-1 $\beta$. Furthermore, the kinetic and the level of IL-8 mRNA expression pattern with Tip110 knockdown and TNF- $\alpha$ treatment was different from TNF- $\alpha$ and IL-1 $\beta$ expression at different time points (Additional file 1: Figure S1A-C) indicating that IL-8 induction by Tip110 knockdown in melanoma cells is more likely independent of autocrine TNF- $\alpha$ and IL- $1 \beta$ regulations. Induction of IL- 8 by Tip110 knockdown was also observed in other tested melanoma cell lines (Additional file 1: Figure S2A-D), while no such effect was observed in the cell lines from other cancer types (Additional file 1: Figure S2E-I) except for non-small-cell lung cancer cells, H1299, but the magnitude of IL-8 induction was relatively small compared to that in melanoma cells with TNF- $\alpha$ treatment (Additional file 1: Figure S2J).

IL-8 mRNA is highly expressed in hypoxic areas of several tumors including malignant melanoma, glioblastoma and ovarian carcinoma [1]. Thus, we examined the expression level of selected cytokines in response to a severe hypoxic condition and Tip110 knockdown. qRT-PCR analysis revealed that hypoxia-induced up-regulation of IL-8 was significantly enhanced by Tip 110 knockdown in melanoma (Fig. 1b). Unlike IL-8, the expression of TNF- $\alpha$ and IL-1 $\beta$ in response to hypoxia and Tip110 knockdown was reduced (Fig. 1c and d). In addition, Tip110 expression was suppressed in the core tumor and presumably severe hypoxic area of mouse bone metastasis tissues obtained by inoculating metastatic melanoma 1205Lu ([3] and Fig. 1e).
Tip110 knockdown in 1205Lu cells increased IL-8 protein expression and stability compared to control transfected cells by using cycloheximide ( $\mathrm{CHX}$ ) chase assay (Fig. 1f). Using ELISA assays, we also detected increased levels of IL- 8 in the conditioned media from Tip110-knockdown 1205Lu cells with and without TNF- $\alpha$ treatment (Fig. 1g). Furthermore, conditioned media of Tip110-knockdown cells significantly increased the invasiveness of melanoma cells (Fig. 1h). These results suggest that Tip110 downregulation in melanoma cells leads to enhanced cell invasion, likely through induction of IL- 8 production and release as a growing number of studies have shown that IL- 8 is associated with tumor cells migration and invasion [1].

\section{Tip110 regulated IL-8 mRNA stability}

The regulation of IL-8 expression involves both transcriptional and posttranscriptional mechanisms [7]. Tip110 knockdown showed modest activation of the IL-8 promoter activity (Fig. 2a) indicating that induction of IL- 8 by Tip110 knockdown does not solely rely on the IL-8 transcriptional activity. IL-8 could also potentially be induced in cancer cells at the level of RNA synthesis and/ or mRNA stability [7]. Considering the role of Tip110 in RNA processing [2], we investigated whether Tip110 knockdown would induce IL-8 through affecting the processing of $I L-8$ gene transcripts and their stability. Using specific primer sets (Fig. 2b), which specifically distinguish IL-8 pre-mRNA and mature mRNA, we found that in contrast to increases of the IL-8 pre-mRNA, the IL-8 mature mRNA was disproportionally increased in si-Tip110transfected cells (Fig. 2c). These results indicate that Tip110 likely regulates IL- 8 mRNA stability rather than IL-8 pre-mRNA synthesis. To further address this possibility, we performed an actinomycin D (ActD) chase assay. The results showed that Tip110 knockdown significantly prolonged half-life of the IL-8 mRNA compared to the control (Fig. 2d and e). Previously, we showed that Tip110 knockdown up-regulated TNF- $\alpha$ mRNA (Fig. 1). Therefore, we examined whether Tip110 would affect TNF- $\alpha$ mRNA stability in a similar fashion to the IL-8. Interestingly, TNF- $\alpha$ mRNA was highly unstable by knockdown Tip110 (Fig. 2f and g), although the initial mRNA level at 0-h time point was much higher.

Since the p38 MAPK signaling pathway is known to play a major role in IL-8 post-transcriptional regulation and mRNA stability [7], we determined the roles of Tip110 knockdown regulation of IL-8 transcript levels. The results showed that treatment of cells with p38 MAPK signaling inhibitor led to significant attenuation of IL-8-up-regulation at the mRNA level in Tip110 knocked down cells (Fig. 2h). These results demonstrated that Tip110 knockdown specifically stabilized and sustained 


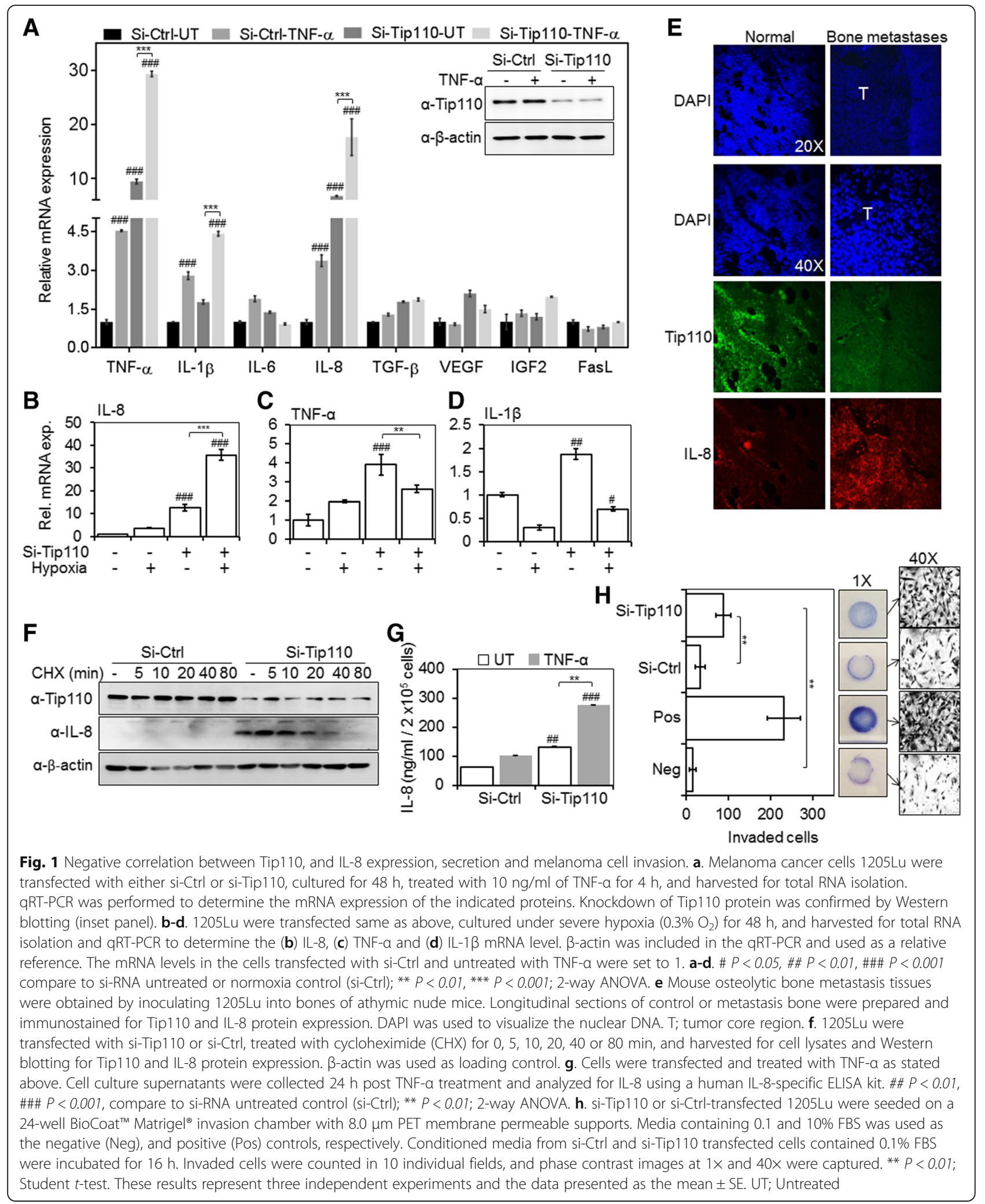




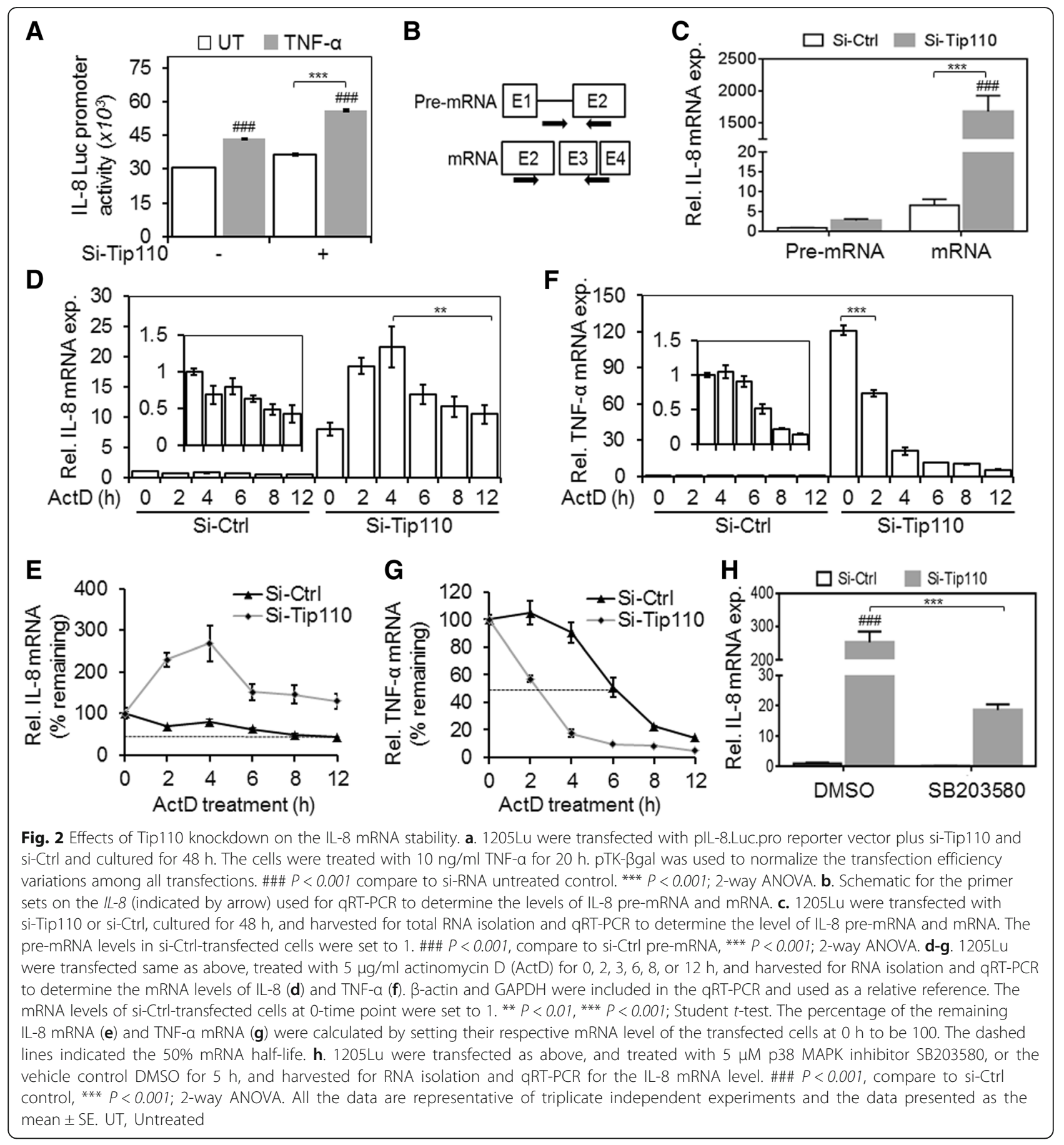

the $I L-8$ gene transcripts, most likely through p38 MAPK signaling.

Tip110 has the ability to recognize and bind to RNA, and early studies have revealed that Tip110 plays a role in the regulation of mRNA synthesis [2]. However, Tip110 knockout in zebrafish can trigger compensatory responses by up-regulating other mRNA synthesis related components [8]. Unexpectedly, in Tip110 knockdown cells, we found an increase in IL- 8 mRNA after short-time exposure to ActD (Fig. 2d and e). We speculate that the unexpected findings may be due to robust and high kinetics rate of IL- 8 mRNA synthesis compared to TNF- $\alpha$ mRNA in the context of a limited amount of Tip110. Indeed, It has been shown in primary human monocytes and macrophages that there is a much stronger and sustained novel type of IL-8 mRNA stabilization compared to TNF- $\alpha$ mRNA [9]. The (AU)-rich sequence in the 3'-UTR of the IL-8 gene has been suggested to 
contribute to its post-transcriptional regulation [10]. Therefore, it is possible that Tip110 targets the 3'-UTR of IL- 8 and regulates its mRNA stability in cancer.

\section{Stage-dependent expression of Tip110 correlated with the clinical outcomes of melanoma patients}

To determine whether Tip110 expression would be correlated with clinical outcomes of melanoma patients, we mined the transcriptomic profiling data and survival of melanoma patients available in the TCGA database. The median follow-up was 28.8 months in the analyzed cohort that was composed of 455 (female $=180$ and male $=289$ ) adult melanoma cancer patients (mean age: 58 years). Of all patients, 417 had no evidence of metastasis $(M=0)$ while 24 patients had melanoma spread from the primary site to other areas of the skin or under the skin, or distant lymph nodes $(M=1)$ (Additional file 1: Figure S3A). The patients were further grouped based on the melanoma stage, primary tumors $(\mathrm{T})$ and regional lymph nodes $(\mathrm{N})$ (Additional file 1: Figure S3C-F). The survival analysis using all patients in the database showed that melanoma cancer patients with high Tip110 expression experienced worse overall survival rates (Fig. 3a). Interestingly, when we ran the survival analysis using patients at "stage 1 " $(n=77)$; which was defined as melanoma that is up to $2 \mathrm{~mm}$ thick, no lymph node involvement and no distal metastasis; the patients with low Tip110 expression experienced significantly worse overall survival rates (Fig. 3b). We further analyzed the overall survival of melanoma patients with respect to IL-8 expression and found no significant difference in the overall survival of either all patients or the patients with "stage 1" tumors (Fig. 3c and d). However, there was an inverse correlation on the overall survival between the Tip110 and IL-8 expression at the "stage 1". Recently, it has been reported that changes in serum IL-8 levels during the immunotherapy treatment correlate with overall survival in patients with metastatic melanoma and non-small-cell lung cancer [11].

The results further demonstrated that TP53 expression, which is found to be regulated by Tip110 [3], at "all stages" was not prognostic in malignant melanoma, while at "stage 1 " low expression of p53 showed poor prognosis similar to the Tip110 in the same patient subpopulation (Fig. 3e and $\mathrm{f}$ ). When we divided the samples into two cohorts according to the TP53 status, we found no significant correlation between TP53 status and Tip110 expression (data not shown). 89\% of all melanoma patients $(n=387)$ in our study expressed wild-type TP53 (Additional file 1: Figure S3B). An early study has shown that an increased level of TP53 protein does not indicate an increased degree of malignancy in melanoma, but rather suggests a more favorable disease progression [12]. In addition, TP53 is also tightly regulated
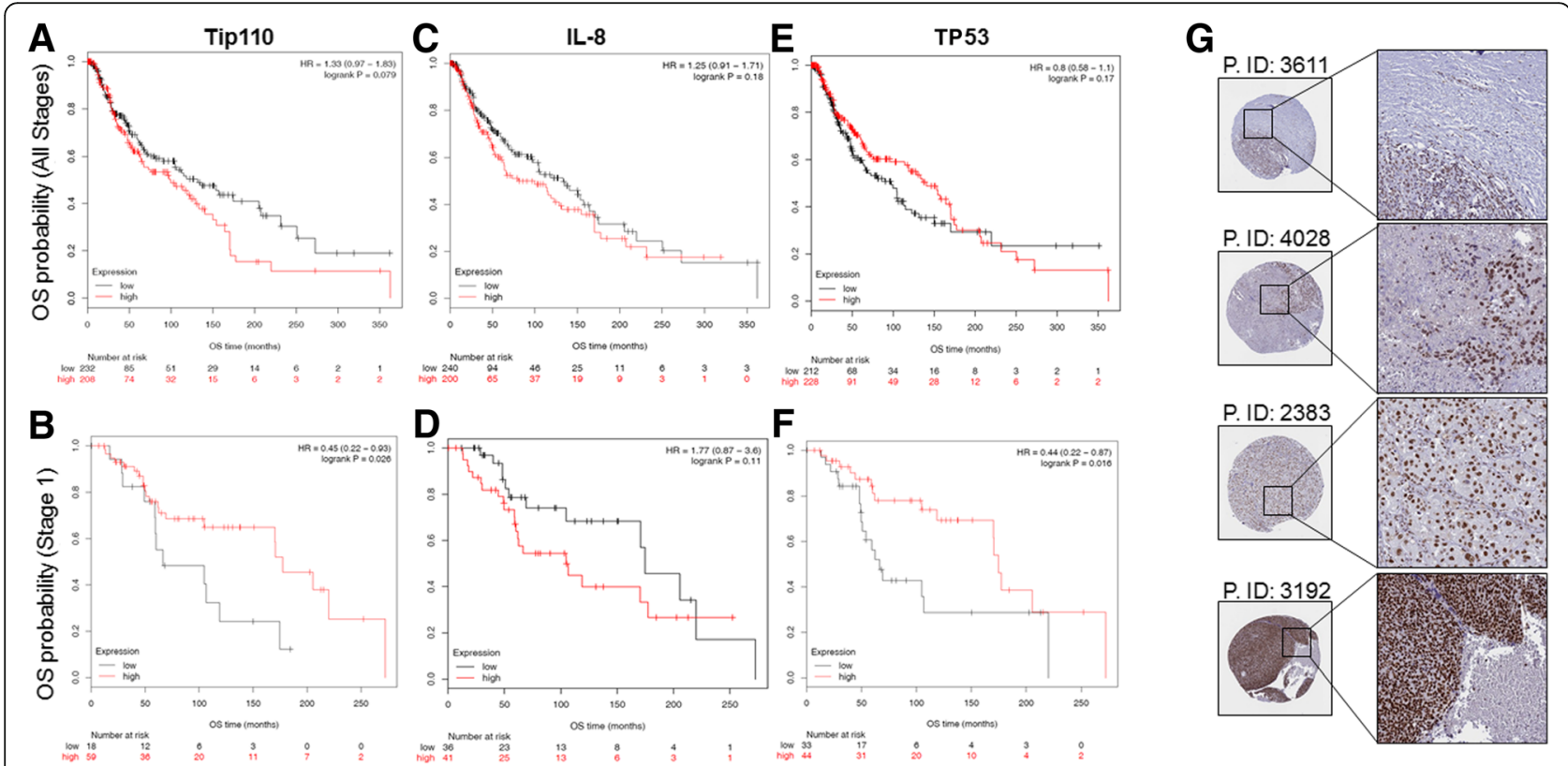

Fig. 3 Correlation between Tip110, IL-8, TP53 expression and clinical outcome in melanoma patients. RNA-Seq data from TCGA were used to assess the correlation between Tip110 expression and the overall survival (OS) probability ( $\mathbf{a}$ and $\mathbf{b}$ ), between IL-8 expression and the OS probability (c and $\mathbf{d}$ ), and between p53 expression and the OS probability (e and $\mathbf{f}$ ). The OS probability was calculated separately for all stages melanoma patients (a, c and $\mathbf{e}$ ) and for stage 1 melanoma patients (b, $\mathbf{d}$ and $\mathbf{f}$ ), which is defined as melanoma that is up to 2 mm thickness (Additional file 1: Figure S3E). HR, hazard ratio. g. Tip110 expression in malignant melanoma cancer tissues. Images credit: Human Protein Atlas, (www.proteinatlas.org) [13]. Images are available at the following URL: v16.proteinatlas.org/humancell. P.ID: patient identification number 
at the translational level through phosphorylation and ubiquitination while the overall survival data were obtained based on the TP53 mRNA level.

When we examined the Tip110 expression in melanoma patients' tissues using the human protein atlas online tool [13], we found several melanoma tumor samples show a high level of Tip110 expression. Interestingly, some patients' samples appeared to have a high degree of heterogeneity in Tip110 expression throughout a tissue section (Fig. 3g, subset). The overall findings suggest the importance of differential expression of Tip110 in melanoma tumorigenesis and its potential prognostic value in patients' clinical outcomes.

\section{Conclusions}

Our findings demonstrated the importance of the differential expression of Tip110 as a prognostic indicator of melanoma tumorigenesis and the potential link to tumor metastasis. Moreover, the roles of Tip110 in regulating the production of IL-8 in melanoma suggest that the target genes or regulators of Tip110 may be suitable for therapeutic intervention.

\section{Additional files}

Additional file 1: Supplementary materials. (ZIP 3620 kb)

\begin{abstract}
Abbreviations
ActD: Actinomycin D; CHX: Cycloheximide; ELISA: Enzyme-linked immunosorbent assay; Fas L: Fas ligand; IGF2: Insulin-like growth factor 2; IL1 $\beta$ : Interleukin 1 beta; IL-6: Interleukin 6; IL-8: Interleukin 8; p38 MAPK: p38 mitogen-activated protein kinases; SART3: Squamous cell carcinoma antigen recognized by T cells 3; TCGA: The cancer genome atlas; TGF-

B1: Transforming growth factor beta 1; Tip110: HIV-1 Tat-interaction protein TNF-a: Tumor necrosis factor-alpha; TP53: Tumor protein 53; VEGF: Vascular endothelial growth factor
\end{abstract}

\section{Acknowledgments}

We are grateful to Dr. Yu-Chieh Wang, Victor Lin and Ashwini Zolekar for their constructive inputs and generously providing melanoma cell lines. We are grateful to Dr. Ann Roman for providing human foreskin fibroblasts. We apologize for being unable to cite several reported studies in this manuscript due to the limited number of references allowed by the journal policy.

\section{Funding}

This work was supported by University of North Texas Health Science Center intramural grant R10077 to K.A.T.

\section{Availability of data and materials}

Data analyzed in the present study are available in the TCGA databases (Additional file 1).

\section{Authors' contributions}

KAT: conceptualized and executed the study, interpreted the data and wrote the MS. BJ: analyzed TCGA data, gave valuable inputs, and helped in editing the MS. YL: shared reagents and gave valuable inputs. KSM: shared reagents, gave valuable inputs, and helped in editing the MS. JH: conceptualized the study, supervised experiments, supported the study and contributed to writing the MS. All the authors approved the submission of the manuscript.
Consent for publication

Not applicable.

\section{Competing interests}

The authors declare that they have no competing interests.

\section{Publisher's Note}

Springer Nature remains neutral with regard to jurisdictional claims in published maps and institutional affiliations.

\section{Author details}

${ }^{1}$ Department of Microbiology, Immunology, and Genetics, University of North Texas Health Science Center, 3500 Camp Bowie Blvd, Fort Worth, TX 76107, USA. ${ }^{2}$ MTA TTK Lendület Cancer Biomarker Research Group, Institute of Enzymology, Magyar Tudósok körútja 2, Budapest 1117, Hungary.

${ }^{3}$ Semmekweis University 2nd Department of Pediatrics, Tűzoltó utca 7-9, Budapest 1094, Hungary. ${ }^{4}$ Division of Endocrinology, Department of Internal Medicine, Indiana University School of Medicine, Indianapolis, IN 46202, USA.

Received: 10 January 2018 Accepted: 31 July 2018

Published online: 17 August 2018

\section{References}

1. Waugh DJ, Wilson C. The interleukin-8 pathway in cancer. Clin Cancer Res. 2008;14:6735-41.

2. Whitmill A, Timani KA, Liu Y, He JJ. Tip110: physical properties, primary structure, and biological functions. Life Sci. 2016;149:79-95.

3. Timani KA, Liu Y, Fan Y, Mohammad KS, He JJ. Tip110 regulates the cross talk between p53 and hypoxia-inducible factor 1alpha under hypoxia and promotes survival of cancer cells. Mol Cell Biol. 2015;35:2254-64.

4. Clark EA, Golub TR, Lander ES, Hynes RO. Genomic analysis of metastasis reveals an essential role for RhoC. Nature. 2000;406:532-5.

5. Cui YQ, Geng Q, Yu T, Zhang FL, Lin HC, Li J, Zhu MX, Liu L, Yao M, Yan MX. Establishment of a highly metastatic model with a newly isolated lung adenocarcinoma cell line. Int J Oncol. 2015;47:927-40.

6. Elias EG, Hasskamp JH, Sharma BK. Cytokines and growth factors expressed by human cutaneous melanoma. Cancers (Basel). 2010;2:794-808.

7. Hoffmann E, Dittrich-Breiholz O, Holtmann H, Kracht M. Multiple control of interleukin-8 gene expression. J Leukoc Biol. 2002;72:847-55.

8. Trede NS, Medenbach J, Damianov A, Hung LH, Weber GJ, Paw BH, Zhou Y, Hersey C, Zapata A, Keefe M, Barut BA, Stuart AB, Katz T, Amemiya CT, Zon $\mathrm{LI}$, Bindereif A. Network of coregulated spliceosome components revealed by zebrafish mutant in recycling factor p110. Proc Natl Acad Sci U S A. 2007;104:6608-13.

9. Mahmoud L, Al-Enezi F, Al-Saif M, Warsy A, Khabar KS, Hitti EG. Sustained stabilization of Interleukin-8 mRNA in human macrophages. RNA Biol. 2014; 11:124-33.

10. Yu Y, Chadee K. The 3'-untranslated region of human interleukin-8 mRNA suppresses IL-8 gene expression. Immunology. 2001;102:498-505.

11. Sanmamed MF, Perez-Gracia JL, Schalper KA, Fusco JP, Gonzalez A, Rodriguez-Ruiz ME, Onate C, Perez G, Alfaro C, Martin-Algarra S, Andueza MP, Gurpide A, Morgado M, Wang J, Bacchiocchi A, Halaban R, Kluger H, Chen L, Sznol M, Melero I. Changes in serum interleukin-8 (IL-8) levels reflect and predict response to anti-PD-1 treatment in melanoma and non-smallcell lung cancer patients. Ann Oncol. 2017;28:1988-95.

12. Florenes VA, Holm R, Fodstad O. Accumulation of p53 protein in human malignant melanoma. Relationship to clinical outcome. Melanoma Res. 1995;5:183-7.

13. Uhlen M, Fagerberg L, Hallstrom BM, Lindskog C, Oksvold P, Mardinoglu A, Sivertsson A, Kampf C, Sjostedt E, Asplund A, Olsson I, Edlund K, Lundberg E, Navani S, Szigyarto CA, Odeberg J, Djureinovic D, Takanen JO, Hober S, Alm T, Edqvist PH, Berling H, Tegel H, Mulder J, Rockberg J, Nilsson P, Schwenk JM, Hamsten M, von Feilitzen K, Forsberg M, Persson L, Johansson F, Zwahlen M, von Heijne G, Nielsen J, Ponten F. Proteomics Tissue-based map of the human proteome. Science. 2015;347:1260419. 